\title{
Développement d'une méthode de dépistage toxicologique large par HPLC/DAD : comparaison de trois méthodes d'extraction du sérum, extraction liquide-liquide et extractions solide-liquide (Bond Elut Certify $^{\circledast}$ et OASIS ${ }^{\circledast}$ MCX)
}

\section{Development of a method of large toxicologi- cal screening by HPLC/DAD : comparaison of three methods of serum extraction, liquid- liquid extraction and solid-liquid extractions (Bond Elut Certify ${ }^{\circledR}$ and OASIS ${ }^{\oplus} M C X$ )}

Sophie GINOLHAC*, Mustapha MOULSMA, Gisèle LARDET, Jean-Jacques VALLON ${ }^{\dagger}$,

Laboratoire de Pharmacotoxicologie et Analyses de Traces, Hôpital Édouard Herriot, place d'Arsonval - LYON

* Auteur à qui adresser la correspondance : Sophie GINOLHAC, Centre International de Recherche sur le Cancer, 150, cours Albert Thomas - 69372 LYON Cedex 08 - Tél : 0472738364 - Fax : 0472738322

(Reçu le 5 janvier 2001 ; accepté le 2 mars 2001)

\section{RÉSUMÉ}

Les auteurs proposent une méthode de dépistage toxicologique large de xénobiotiques médicamenteux dans le sérum faisant appel à une étape préanalytique nécessaire à une séparation par chromatographie liquide haute performance (HPLC) et une détection UV à barrettes de diode. Cette méthode permet la détection et la quantification de nombreuses molécules de polarité variée.

Trois types d'extraction sont comparées : extraction liquide-

\section{SUMMARY}

The authors propose a broad screening method of toxic molecules in blood comprising a serum extraction, a high phase liquid chromatographic separation (HPLC) and an $U V$ diode array detection. This method estimates the quantity of different molecular compounds of varied polarity. Three extractions methods were compared : liquid-liquid extraction, solid-phase extraction on silica columns (BOND ELUT CERTIFY $Y^{\circledR}$ ) and solid-phase extraction on copolymer 
liquide, extraction solide-liquide sur colonne de silice (BOND ELUT CERTIFY ${ }^{\circledR}$ ) et extraction solide-liquide sur colonne de copolymère (OASIS $\left.{ }^{\circledR} M C X\right)$, afin d'évaluer la meilleure technique pour l'objectif recherché. L'analyse chromatographique est réalisée sur HPLC/DAD, HPI100 équipé d'une colonne hypersil BDS C18, 3X125 mm. La phase mobile est un tampon phosphate 0,1M pH 5,6.

L'extraction sur colonne OASIS ${ }^{\circledR}$ MCX donne, en moyenne, pour l'ensemble des molécules analysées, les rendements d'extraction les plus importants avec une bonne répétabilité. Seuls les anti-inflammatoires non stéroïdiens sont mal extraits par cette technique mais leurs concentrations thérapeutiques étant importantes, leur détection est possible. Outre ses bons rendements, la colonne OASIS ${ }^{\circledR}$ MCX permet une extraction plus rapide, une moindre utilisation de solvant et de sérum que les autres techniques.

Cette colonne est donc bien adaptée aux dépistages toxicologiques larges à partir de sérum.

\section{MOTS-CLÉS}

Dépistage toxicologique, extraction phase solide.

\section{Introduction}

Les services d'urgences des centres hospitaliers universitaires sont confrontés à un grand nombre d'intoxications aiguës. Leurs causes sont très diverses et sont dues le plus souvent à des médicaments (1) : surdosages médicamenteux volontaires ou accidentels, abus de drogues et/ou d'alcool mais aussi expositions à des produits industriels ou ménagers. Les toxiques animaux et végétaux sont plus rarement en cause sous nos climats (2).

L'analyse toxicologique permet de confirmer ou de réfuter l'hypothèse diagnostique et peut contribuer à évaluer le pronostic et à surveiller le traitement de certaines intoxications (3).

Dans la plupart des dépistages toxicologiques décrits dans la littérature, l'extraction des composés à partir des milieux biologiques est réalisée en milieu basique, permettant la séparation et l'identification de molécules essentiellement basiques et neutres (4-5-6-7-8).

Peu d'auteurs travaillent sur les molécules acides (9). Certains décrivent l'extraction ciblée à une classe pharmacologique comme les benzodiazépines ou les antidépresseurs (10-11-12). Le Remedi est un appareil entièrement automatisé permettant l'extraction, la séparation et l'identification en ligne des différentes molécules présentes dans un échantillon, mais seules les molécules basiques et neutres sont extraites avec cette technique (13-14-15-46). Ces molécules sont celles le plus largement rencontrées en toxicologie clinique mais des molécules acides sont parfois impliquées dans les tentatives de suicide comme les antiinflammatoires non stérö̈diens, les salicylés, l'acide valproïque... Peu d'articles décrivent un dépistage toxi- columns (OASIS $\left.{ }^{\circledR} M C X\right)$. The chromatographic analysis was carried out on a HPLC/DAD HPI100 analyser with a hypersil BDS column C18 $3 X 125 \mathrm{~mm}$ and the mobile phase was phosphate buffer 0,1M pH 5,6.

The column OASIS ${ }^{\circledR}$ MCX generally gave the best results and was the most reliable. However, the non steroidal antiinflammatory drugs were poorly extracted with this column but as their therapeutic concentrations are high, their detection was still possible. In addition to its good resolution, the column OASIS ${ }^{\circledR}$ MCX allows a faster extraction, and uses less solvent and serum than the other techniques. This column is thus well adapted to broad toxicological screening starting from serum.

\section{KEY-WORDS}

Toxicological screening, solid phase extraction.

cologique large permettant l'extraction de composés acides, basiques et neutres (17-18-19)

Le service des urgences de l'hôpital Edouard HERRIOT à Lyon est le plus important de la région. Le laboratoire de toxicologie de cet hôpital a mis en place un dépistage large, faisant appel à plusieurs méthodologies analytiques complémentaires dont la chromatographie en phase liquide qui permet l'identification et la quantification de molécules basiques et neutres. Le travail présenté ici a pour objectif d'élargir cette analyse aux molécules acides, en évaluant un nouveau support d'extraction. Afin d'évaluer la meilleure extraction possible pour l'objectif recherché, plusieurs types d'extraction sont comparés : extraction liquide-liquide, extraction solide-liquide sur colonne de silice greffée (BOND ELUT CERTIFY ${ }^{\circledR}$ ), et extraction solide-liquide sur colonne de copolymère greffé (OASIS ${ }^{\circledR}$ MCX).

77 molécules sont extraites par ces trois méthodes : 12 antidépresseurs tricycliques et apparentés, 8 inhibiteurs de la recapture de la sérotonine, 16 neuroleptiques, 19 benzodiazépines et apparentés, 8 anti-inflammatoires non stéroïdiens et 13 molécules diverses. L'objectif est de travailler sur un ensemble de molécules présentant une gamme de polarité variée, des structures diverses et régulièrement rencontrées en toxicologie clinique. $\mathrm{La}$ majorité des molécules sélectionnées sont basiques et neutres et donc déjà identifiées et quantifiées par la méthode de dépistage large utilisées en routine au laboratoire. Ces molécules permettront d'évaluer les extractions solide-liquide par rapport à l'extraction liquide-liquide, technique de routine du laboratoire. Les molécules acides sont représentées dans cette étude par les anti-inflammatoires non stéroïdiens, permettant d'élargir le dépistage toxicologique à une gamme plus 
large de molécules. Seuls quelques principes actifs ont été étudiés dans ce travail mais ces techniques d'extraction peuvent s'appliquer à un nombre beaucoup plus important de molécules et de classes thérapeutiques.

\section{Matériel et méthodes}

\section{Matériel et réactifs}

Les colonnes SPE étudiées sont les colonnes BOND ELUT CERTIFY ${ }^{\circledR}$ (BEC) C18

$10 \mathrm{ml} / 130 \mathrm{mg}$ (Analytichem International, Harbor City, California) et les colonnes OASIS ${ }^{\circledR}$ MCX $3 \mathrm{ml} / 60 \mathrm{mg}$ (Waters).

Tous les réactifs utilisés sont de qualité chromatographique. Ils proviennent des laboratoires Merck (Darmstat, Germany) excepté le chlorobutane (Fluka, Buchs, switzerland), la triéthylamine, et l'ammoniac $28 \%$ (Prolabo, Fontenay-sous-Bois, France).

Tous les principes actifs utilisés pour préparer les solutions de travail sont de qualité pharmaceutique. Ils se présentent sous forme de poudres distribuées par Sigma Chemical Co (St Louis, MO, U.S.A.) ou de solutions distribuées par Promochem (Radian). Enfin, certains proviennent de spécialités pharmaceutiques (comprimés, gélules ou solutions injectables) délivrées par la pharmacie de l'hôpital Edouard Herriot.

Les solutions de travail sont préparées à une concentration de 40,50 ou $100 \mathrm{mg} / \mathrm{l}$ en fonction de la concentration de la solution mère ou du dosage initial en principe actif de la forme pharmaceutique. Les solutions de proguanil et de milnacipran sont à $87 \mathrm{mg} / \mathrm{l}$. Les solutions d'anti-inflammatoires non stéroïdiens sont à $1 \mathrm{~g} / \mathrm{l}$ excepté pour le fénoprofène $(1,2 \mathrm{~g} / \mathrm{l})$ et le diflunisal $(5 \mathrm{~g} / \mathrm{l})$.

Les pools de sérum négatifs sont préparés à partir de sérums chauffés 60 minutes à $56^{\circ} \mathrm{C}$ pour inactiver les virus. Ils sont testés après extraction liquide-liquide en HPLC/DAD (méthode de routine).

\section{Instrumentation}

La séparation est réalisée à l'aide d'un chromatographe en phase liquide série 1100 (Hewlett Packard, France) équipé d'un détecteur à barrette de diodes (DAD) et d'une colonne Hypersil BDS C18 de $3 \mathrm{~mm}$ de diamètre et de $125 \mathrm{~mm}$ de long. La granulométrie de la phase est de $3 \mu \mathrm{m}$ de diamètre. Une précolonne Superspher 100 RP $1810 \times 2 \mathrm{~mm}$ est placée en tête de colonne. La température de l'enceinte thermostatée est de $27^{\circ} \mathrm{C}$. La phase mobile est un mélange de $700 \mathrm{ml}$ d'un tampon phosphate $0,1 \mathrm{M} \mathrm{pH} 5,6$ contenant 6,5 pour mille de triéthylamine, avec $300 \mathrm{ml}$ d'acétonitrile. L'élution est réalisée en mode isocratique à un débit de $0,7 \mathrm{ml} / \mathrm{min}$.
$20 \mu \mathrm{l}$ d'échantillon sont injectés dans la colonne et l'analyse chromatographique dure 20 minutes. Les longueurs d'onde d'acquisition utilisées sont 226, 240, $270,320 \mathrm{~nm}$.

Le logiciel de traitement des données est le logiciel HP Chemstation for LC, version A.06.01 (403), 19901998. L'analyse spectrale est automatique à l'aide d'une bibliothèque réalisée par le laboratoire.

\section{Méthodes}

\section{Extraction liquide/liquide}

- En milieu basique : à un $\mathrm{ml}$ de sérum sont rajoutés successivement $100 \mu \mathrm{l}$ d'ammoniaque à $28 \%(\mathrm{pH}=9)$ et $3 \mathrm{ml}$ de chlorobutane. Le tube est agité 6 minutes à l'aide d'un système d'agitation horizontale. Après centrifugation, la phase organique est récupérée et $3 \mathrm{ml}$ de chlorobutane sont à nouveau ajoutés au sérum. L'opération est donc répétée deux fois et les $6 \mathrm{ml}$ de phase organique ainsi obtenues sont mélangées et évaporées (fraction basique). Cette technique correspond à celle utilisée en routine dans notre laboratoire.

- En milieu acide : la procédure est identique à la précédente mais le milieu est acidifié par $100 \mu \mathrm{l}$ d'acide chlorhydrique $2 \mathrm{~N}$ (fraction acide).

\section{Extraction solide-liquide sur la BEC (17)}

La colonne est conditionnée par $2 \mathrm{ml}$ de méthanol $(\mathrm{MEOH})$ et $2 \mathrm{ml}$ de tampon phosphate $0,1 \mathrm{M}(\mathrm{pH}=6)$. Un $\mathrm{ml}$ de sérum est dilué dans $6 \mathrm{ml}$ de tampon phosphate. Après agitation au vortex, le mélange est passé à travers la colonne grâce à l'utilisation d'un système Vac Elut $^{\circledR}$ (Varian). La colonne est lavée par $1 \mathrm{ml}$ d'eau et acidifiée à $\mathrm{pH} 3,3$ par $0,5 \mathrm{ml}$ d'une solution d'acide acétique $0,01 \mathrm{M}$. La colonne est ensuite séchée $2 \mathrm{~min}, 50 \mu \mathrm{l}$ de MEOH sont passés à travers la colonne puis celle-ci est séchée à nouveau 2 minutes. L'échantillon est élué dans un premier temps par $4 \mathrm{ml}$ d'un mélange acétone/chloroforme (50/50) (fraction acide) puis par $2 \mathrm{ml}$ d'acétate d'éthyle ammoniaqué ( $2 \%$ ) (fraction basique).

\section{Extraction solide-liquide sur l'OASIS ${ }^{\circledR} M C X$}

Plusieurs essais préalables de lavage et d'élution ont été réalisés. Le protocole d'extraction de la BEC a notamment été réalisé sur l'OASIS ${ }^{\circledR}$ mais le chromatogramme était très chargé. Le protocole suivant a été retenu.

La colonne est conditionnée par $2 \mathrm{ml}$ de méthanol et $2 \mathrm{ml}$ d'eau stérile. Un ml de sérum est passé à travers la colonne grâce à l'utilisation d'un Vac Elut ${ }^{\circledR}$. Puis la colonne est lavée et acidifiée par $2 \mathrm{ml}$ d'un mélange d'acide chlorhydrique $0,1 \mathrm{~N}$ et de $\mathrm{MEOH}(80 / 20)$ $(\mathrm{pH}=1)$. L'échantillon est ensuite élué par $2 \mathrm{ml}$ d'un mélange méthyl-ter-buthyl-éther (MTBE) et $\mathrm{MEOH}$ 
(90/10) (fraction acide) puis par $2 \mathrm{ml}$ d'un mélange MTBE/MEOH (90/10) contenant $2 \%$ d'ammoniac (fraction basique).

Pour toutes les méthodes d'extraction, les différentes fractions obtenues sont évaporées séparément dans un Speed $\mathrm{Vac}^{\circledR}$ (Savant Instrument INC, New York) et reprises chacune par $60 \mu \mathrm{l}$ d'un mélange acétonitrile/eau (30/70). Les extraits repris sont vortexés 30 secondes pour solubiliser le maximum de molécules puis analysés séparément par HPLC/DAD.

Pour analyser un prélèvement provenant d'un service de l'hôpital, $10 \mu \mathrm{l}$ d'étalon interne, le désalkylflurazépam $(25 \mathrm{mg} / \mathrm{l})$ sont ajoutés à un $\mathrm{ml}$ de sérum avant le début de l'extraction, quelque soit la méthode utilisée. Le désalkylflurazépam est le métabolite du loflazépate d'éthyle $\left(\operatorname{victan}^{\circledR}\right)$. Si ce métabolite est présent dans le sérum analysé, il modifie la quantité d'étalon interne dans l'échantillon, perturbant ainsi le dosage. Dans ce cas, l'extraction est refaite sans ajouter de désalkylflurazépam et la quantification est réalisée sans étalon interne.

\section{Calcul du rendement d'extraction.}

Les solutions de travail sont diluées au centième : la concentration finale des analytes dans l'échantillon est donc, selon les cas de 0,$4 ; 0,5$ ou $1 \mathrm{mg} / \mathrm{l}$ excepté pour le milnacipran et le proguanil $(0,9 \mathrm{mg} / \mathrm{l})$. Pour les antiinflammatoires non stéroïdiens (dont le diflunisal), les concentrations finales sont de $0,5 \mathrm{mg} / \mathrm{l}$ excepté pour le fénoprofène $(0,6 \mathrm{mg} / \mathrm{l})$.

Les molécules sont regroupées dans différents mélanges. Pour calculer le rendement d'extraction, deux tubes $\mathrm{T} 1$ et $\mathrm{T} 2$ sont préparés : T1 contient $900 \mu \mathrm{l}$ de sérum et $100 \mu \mathrm{l}$ d'un mélange $\mathrm{A}$ et $\mathrm{T} 2$ contient $1 \mathrm{ml}$ de sérum. $\mathrm{T} 1$ et $\mathrm{T} 2$ sont extraits en même temps. Après évaporation des différentes fractions, $10 \mu \mathrm{l}$ de désalkylflurazépam $(25 \mathrm{mg} / \mathrm{l})$ sont ajoutés dans T1. Dans le tube $\mathrm{T} 2$, on ajoute $10 \mu \mathrm{l}$ de désalkylflurazépam $(25 \mathrm{mg} / \mathrm{l})$ et $100 \mu \mathrm{l}$ de mélange $\mathrm{A}$. Ces petits volumes sont évaporés, les résidus sont repris par $60 \mu \mathrm{l}$ d'acétonitrile/eau (30/70) et analysés séparément par HPLC/DAD. Pour chaque extrait, le rapport de l'aire du pic de la molécule sur celle du pic du désalkylflurazépam est calculé (R1 pour le tube $\mathrm{T} 1$ et $\mathrm{R} 2$ pour le tube T2). Le rendement correspond au rapport de R1/R2 multiplié par 100 .

Le désalkylflurazépam sert ici d'étalon externe à l'extraction, afin de s'affranchir des variations dues éventuellement à l'injection.

Les rendements sont comparés par analyse de variance monofactorielle. Les données étant non appariées, nous avons comparé les variances pour vérifier leur homogénéité par le test de Cochran. Seuls les rendements ayant des variances homogènes ont été comparées. Les diffé- rences sont dites statistiquement significatives quand $\mathrm{p}<0,05(20-21)$

\section{Résultats}

Les tableaux I à VI résument les résultats obtenus.

Tous les tableaux ci-dessous donnent le rendement moyen d'extraction avec l'écart standard à la moyenne (entre parenthèses) et le coefficient de variation des molécules étudiées pour les trois types d'extraction. Les moyennes ont été calculées sur 4 valeurs. Les rendements d'extraction ont été comparés par analyse de variance monofactorielle après vérification de l'homogénéité des variances par le test de Cochran. Le seuil de signification est fixé à 0,05 .

\section{Les antidépresseurs (Tableau I)}

La colonne OASIS ${ }^{\circledR}$ MCX donne, en moyenne pour l'ensemble des molécules testées, des rendements d'extraction statistiquement supérieurs à ceux de l'extraction liquide/liquide (ELL) et de l'extraction BEC ( $89 \%, 80 \%$ et $78 \%$ respectivement).

Cependant, dans le cas de la dosulépine, le rendement de l'extraction liquide-liquide est significativement plus important qu'avec les deux autres techniques. L'amoxapine est significativement mieux extraite par la BEC que par l'OASIS ${ }^{\circledR}$ ou que par l'ELL.

Pour toutes les autres molécules testées, l'OASIS ${ }^{\circledR}$ donne des rendements d'extraction supérieurs à ceux obtenus par les deux autres techniques notamment pour la maprotiline.

La répétabilité est meilleure avec la technique OASIS ${ }^{\circledR}$ qu'avec les deux autres extractions.

\section{Les inhibiteurs de la recapture de la sérotonine (IRS) (Tableau II)}

Les inhibiteurs de la recapture de la sérotonine sont mieux extraits, en moyenne, par l'OASIS ${ }^{\circledR}$ MCX (rendement de $92 \%)$ que par la BEC $(71 \%)$ ou par l'ELL (74\%). Les résultats sont significatifs pour la fluoxétine, la norfluoxétine et la fluvoxamine. Seule la venlafaxine semble mieux extraite par la BEC sans que cela soit significatif.

La technique OASIS ${ }^{\circledR}$ présente les coefficients de variation $(\mathrm{CV})$ les plus faibles et donne donc la meilleure répétabilité.

\section{Les neuroleptiques (Tableau III)}

L'extraction OASIS ${ }^{\circledR}$ a donné, en moyenne, pour l'ensemble des molécules testées des rendements supé- 
Tableau I : Rendements d'extraction des antidépresseurs.

\begin{tabular}{|c|cc|c|cc|c|cc|c|}
\hline & \multicolumn{3}{|c|}{ ELL } & \multicolumn{3}{c|}{ OASIS, fraction basique } & \multicolumn{3}{c|}{ BEC, fraction basique } \\
\cline { 2 - 9 } Antidépresseurs & M \% & (ESM) & CV & M \% & (ESM) & CV & M \% & (ESM) & CV \\
\hline Amitriptyline & $\mathbf{8 6}$ & $(6)$ & 15 & $\mathbf{9 2}$ & $(1)$ & 1 & $\mathbf{8 0}$ & $(5)$ & 12 \\
Amoxapine & $\mathbf{6 9}^{\mathbf{B}}$ & $(2)$ & 5 & $\mathbf{7 8}$ & $(2)$ & 5 & $\mathbf{9 0}$ & $(10)$ & 22 \\
Carpipramine & $\mathbf{7 5}$ & $(10)$ & 25 & $\mathbf{8 9}$ & $(3)$ & 7 & $\mathbf{7 1}$ & $(20)$ & 55 \\
Clomipramine & $\mathbf{9 0}$ & $(9)$ & 20 & $\mathbf{9 5}$ & $(2)$ & 4 & $\mathbf{7 7}$ & $(9)$ & 23 \\
Désipramine & $\mathbf{7 2}$ & $(7)$ & 19 & $\mathbf{9 2}$ & $(3)$ & 7 & $\mathbf{7 8}$ & $(16)$ & 40 \\
Dosulépine & $\mathbf{1 0 0}$ & $(3)$ & 5 & $\mathbf{8 5}$ & $(5)$ & 12 & $\mathbf{7 7}$ & $(6)$ & 17 \\
Doxépine & $\mathbf{8 3}$ & $(4)$ & 10 & $\mathbf{9 0}$ & $(2)$ & 3 & $\mathbf{8 7}$ & $(12)$ & 29 \\
Imipramine & $\mathbf{8 2}$ & $(6)$ & 14 & $\mathbf{9 2}$ & $(4)$ & 9 & $\mathbf{8 5}$ & $(16)$ & 38 \\
Maprotiline & $\mathbf{8 2}$ & $(10)$ & 24 & $\mathbf{9 2}$ & $(4)$ & 9 & $\mathbf{6 0}$ & $(10)$ & 32 \\
Miansérine & $\mathbf{7 1}$ & $(7)$ & 20 & $\mathbf{8 3}$ & $(5)$ & 13 & $\mathbf{7 0}$ & $(5)$ & 14 \\
Norclomipramine & $\mathbf{7 9}$ & $(9)$ & 24 & $\mathbf{9 2}$ & $(1)$ & 3 & $\mathbf{8 4}$ & $(11)$ & 26 \\
Nortriptylline & $\mathbf{7 5}$ & $(8)$ & 21 & $\mathbf{9 2}$ & $(1)$ & 3 & $\mathbf{8 2}$ & $(6)$ & 15 \\
\hline
\end{tabular}

$\mathrm{L}$ : résultat significatif par rapport à la technique liquide-liquide.

$\mathrm{B}$ : résultat significatif par rapport à la technique BEC.

$\mathrm{O}$ : résultat significatif par rapport à la technique OASIS.

Tableau II : Rendements d'extraction des inhibiteurs de la recapture de la sérotonine.

\begin{tabular}{|c|c|c|c|c|c|c|c|c|c|}
\hline \multirow{2}{*}{$\begin{array}{l}\text { Inhibiteurs de la } \\
\text { recapture de la } \\
\text { sérotonine }\end{array}$} & \multicolumn{3}{|c|}{ ELL } & \multicolumn{3}{|c|}{ OASIS, fraction basique } & \multicolumn{3}{|c|}{$\mathrm{BEC}$, fraction basique } \\
\hline & M\% & $(\mathrm{ESM})$ & $\mathrm{CV}$ & M\% & $(\mathrm{ESM})$ & $\mathrm{CV}$ & M \% & $(\mathrm{ESM})$ & $\mathrm{CV}$ \\
\hline Citalopram & 82 & (9) & 21 & 90 & (1) & 2 & 88 & (2) & 5 \\
\hline Fluoxétine & $61^{\circ}$ & (9) & 28 & $95^{\mathrm{L} . \mathrm{B}}$ & (1) & 3 & $65^{\circ}$ & (7) & 22 \\
\hline Fluvoxamine & $62^{\circ}$ & (4) & 12 & $86^{\mathrm{L} . \mathrm{B}}$ & (3) & 6 & $64^{\circ}$ & (6) & 20 \\
\hline Milnacipran & 77 & (12) & 32 & 90 & (3) & 6 & 87 & (4) & 10 \\
\hline Norfluoxétine & $62^{\circ}$ & (8) & 26 & $97^{\text {L.B }}$ & (3) & 5 & $\mathbf{5 8}^{\circ}$ & (5) & 18 \\
\hline Paroxétine & 84 & (14) & 32 & 89 & (2) & 4 & 63 & (6) & 19 \\
\hline Sertraline & 87 & (7) & 15 & 107 & (4) & 7 & 71 & $(28)$ & 78 \\
\hline Venlafaxine & 85 & (5) & 11 & 83 & (4) & 10 & 87 & (2) & 4 \\
\hline
\end{tabular}

L : résultat significatif par rapport à la technique liquide-liquide.

$\mathrm{B}$ : résultat significatif par rapport à la technique BEC.

$\mathrm{O}$ : résultat significatif par rapport à la technique OASIS.

rieurs à l'extraction liquide-liquide et à l'extraction par la BEC ( $90 \%, 82 \%$ et $73 \%$ respectivement).

Cependant dans le cas de la thioridazine, l'ELL est statistiquement plus performante que l'OASIS ${ }^{\circledR}$ et que la BEC.

Les rendements d'extraction de la BEC sont en général plus faibles que ceux de l'OASIS ${ }^{\circledR}$ ou de l'extraction liquide-liquide, même si les résultats ne sont pas toujours statistiquement significatifs.

Les coefficients de variation des rendements d'extraction sont plus importants avec la BEC qu'avec l'extraction liquide-liquide ou qu'avec l'OASIS ${ }^{\circledR}$. 
Tableau III : Rendements d'extraction des neuroleptiques.

\begin{tabular}{|c|c|c|c|c|c|c|c|c|c|}
\hline \multirow[b]{2}{*}{ Neuroleptiques } & \multicolumn{3}{|c|}{ ELL } & \multicolumn{3}{|c|}{ OASIS, fraction basique } & \multicolumn{3}{|c|}{$\mathrm{BEC}$, fraction basique } \\
\hline & M\% & (ESM) & $\mathrm{CV}$ & $\mathrm{M} \%$ & (ESM) & $\mathrm{CV}$ & M \% & $(\mathrm{ESM})$ & $\mathrm{CV}$ \\
\hline Alimémazine & 65 & (11) & 34 & 80 & (7) & 17 & 71 & (2) & 6 \\
\hline Amisulpride & 82 & (2) & 6 & 92 & (5) & 11 & 84 & (6) & 13 \\
\hline Clozapine & $77^{\circ}$ & (1) & 4 & $84^{L}$ & (2) & $\because 4$ & 79 & (2) & 6 \\
\hline Norclozapine & $56^{\circ}$ & (6) & 22 & $79^{L}$ & (2) & 5 & 68 & (3) & 7 \\
\hline Cyamémazine & 86 & (1) & 3 & 95 & (8) & 16 & 78 & (6) & 15 \\
\hline Dropéridol & 87 & (2) & 5 & 91 & (4) & 10 & 89 & (2) & 5 \\
\hline Halopéridol & 95 & (3) & 6 & 88 & (4) & 10 & 77 & (11) & 28 \\
\hline Lévomépromazine & $\mathbf{8 3}^{\mathbf{B}}$ & (2) & 4 & $\mathbf{7 5}^{\mathrm{B}}$ & (5) & 12 & $65^{\mathrm{L} .0}$ & (2) & 8 \\
\hline Loxapine & 82 & (9) & 21 & 89 & (2) & 5 & 77 & (7) & 18 \\
\hline Pipampérone & 90 & (4) & 9 & 101 & (7) & 14 & 88 & (2) & 5 \\
\hline Propériciazine & 84 & (6) & 15 & 90 & (2) & 6 & 82 & (5) & 12 \\
\hline Rispéridone & 78 & (6) & 16 & 89 & (4) & 10 & 81 & (1) & 2 \\
\hline Thioridazine & $128^{\text {B.o }}$ & (16) & 25 & $71^{L}$ & (11) & 32 & $48^{\mathrm{L}}$ & (8) & 32 \\
\hline Tropaténine & 86 & (3) & 7 & 93 & (3) & 6 & 68 & (13) & 38 \\
\hline Zuclopentixol & $82^{\circ}$ & (5) & 12 & $104^{\text {L.B }}$ & (5) & 9 & $70^{\circ}$ & (9) & 27 \\
\hline Metzuclopentixol & $51^{\circ}$ & (7) & 23 & $113^{L . B}$ & (5) & 9 & $48^{\circ}$ & (6) & 25 \\
\hline
\end{tabular}

$\mathrm{L}$ : résultat significatif par rapport à la technique liquide-liquide.

$\mathrm{B}$ : résultat significatif par rapport à la technique BEC.

$\mathrm{O}$ : résultat significatif par rapport à la technique OASIS.

\section{Les benzodiazépines. (Tableau IV)}

\section{Fraction acide et fraction basique}

Dans l'extraction solide-liquide, certaines benzodiazépines sont éluées majoritairement dans la fraction acide et d'autres dans la fraction basique.

- Pour l'OASIS ${ }^{\circledR}$ MCX, les molécules éluées dans la fraction acide sont le clobazam et le norclobazam, le clonazépam, le lorazépam, le lormétazépam et le témazépam.

- Pour la BEC, ces molécules sont les mêmes que pour l'OASIS ${ }^{\circledR}$ ainsi que le diazépam, l'estazolam, le nitrazépam, l'oxazépam, et le triazolam.

- Certaines moléculess se retrouvent en proportion équivalente dans les fractions acide et basique. Leur rendement d'extraction est donc d'environ $50 \%$ dans chaque fraction :

$\square$ Pour l'OASIS $^{\circledR}$ : l'oxazépam, le clonazépam et le témazépam

$\square$ Pour la BEC : L'alprazolam, le bromazépam, l'estazolam, le N-desméthyldiazépam (NDD), le nitrazépam et le triazolam.
Les rendements de ces molécules sont donc relativement faibles dans chacune des fractions.

\section{Comparaison des rendements d'extraction}

Pour l'ensemble des molécules testées, l'OASIS ${ }^{(}$ donne en moyenne des résultats statistiquement meilleurs que l'ELL et que la BEC (respectivement $83 \%, 77 \%$ et $71 \%$ ).

Cependant, le témazépam est significativement mieux extrait par l'ELL que par l'OASIS ${ }^{\circledR}$ ou la BEC.

La BEC donne des rendements significativement meilleurs pour le clonazépam et l'oxazépam.

Il faut également noter que la zopiclone est très mal extraite par l'extraction liquide-liquide.

\section{Répétabilité des rendements d'extraction}

Pour la majorité des molécules étudiées, la répétabilité de l'extraction OASIS ${ }^{\circledR}$ est meilleure que celle des autres techniques (CV plus faibles). 


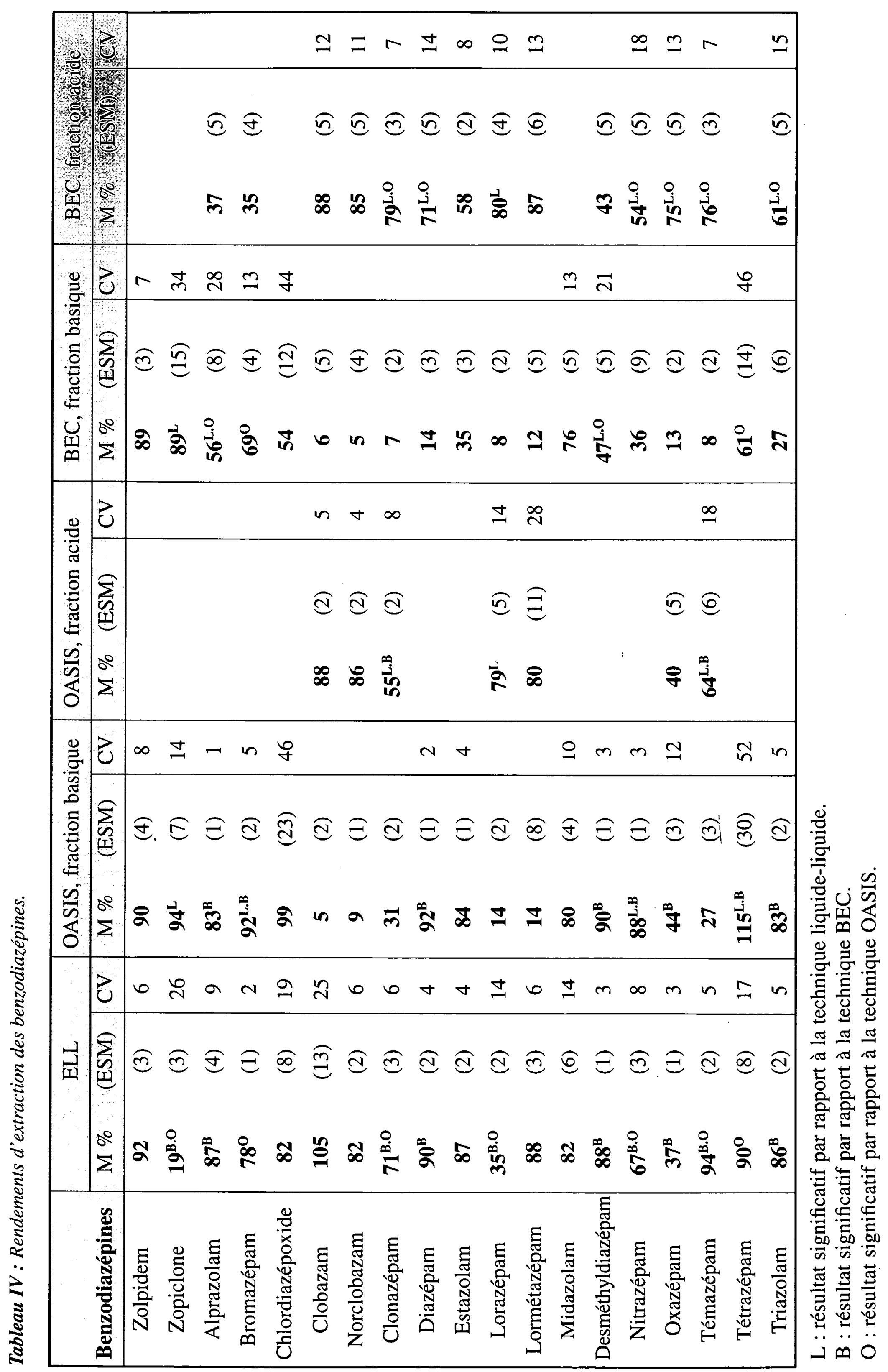




\section{Les AINS. (Tableau V)}

Pour les 8 molécules étudiées dans cette classe pharmacologique, les rendements moyens d'extraction sont beaucoup plus importants avec la technique d'extraction liquide-liquide $(85 \%)$ qu'avec les deux extractions solide-liquide $(20 \%$ pour la BEC et $38 \%$ pour l'OASIS $\left.{ }^{\circledR}\right)$. Les résultats sont significativement meilleurs avec l'OASIS ${ }^{\circledR}$ qu'avec la BEC excepté pour l'indométacine et le piroxicam. En effet, le piroxicam n'est pas extrait sur la colonne OASIS ${ }^{\circledR}$ si le MTBE est utilisé comme solvant d'élution.

La BEC n'extrait pas ou très mal l'acide niflumique, le diflunisal, le fénoprofène et le naproxène.

La répétabilité de l'extraction liquide-liquide est bonne contrairement aux deux autres techniques d'extraction solide-liquide.

\section{Les molécules diverses. (Tableau VI)}

La noramidopyrine est mal extraite par les trois types d'extractions. Le proguanil n'est pas extrait par l'OASIS $^{\circledR}$ et le rendement est faible avec la technique liquide-liquide. La BEC donne une mauvaise repétabilité d'extraction pour la méfloquine. Le propranolol est mieux extrait par l'OASIS ${ }^{\circledR}$ que par la BEC et la technique liquide-liquide.

Enfin, l'extraction liquide-liquide donne des rendements statistiquement plus faibles pour le dilthiazem que les extractions solide-liquide.

\section{Discussion}

Cette étude a été réalisée exclusivement sur du sérum mais d'autres milieux auraient pu être testés comme les urines et le sang total.

Les plasmas vierges utilisés présentent des tracés chromatographiques différents en fonction du type d'extraction réalisée : l'ELL donne les tracés les plus propres alors que l'OASIS ${ }^{\circledR}$ donne les tracés les plus chargés. Cependant, pour l'ensemble des molécules testées, l'extraction OASIS ${ }^{\circledR}$ donne les résultats les plus intéressants : les rendements sont souvent supérieurs ou équivalents aux deux autres extractions. La BEC donne la moins bonne répétabilité d'extraction par rapport aux deux autres techniques étudiées.

Certaines benzodiazépines sont retenues différemment sur les deux colonnes.

Ces différences peuvent s'expliquer par le $\mathrm{pH}$ d'élution qui est de 3,3 pour la BEC et de 1 pour l'OASIS ${ }^{\circledR}$ et par la nature de la phase stationnaire, différente dans chaque colonne. Deux types d'interactions interviennent dans la rétention des composés pour la BEC et l'OASIS $^{\circledR}$. La BEC est composée de silice greffée avec des groupements $\mathrm{C} 18$, responsables d'interactions hydrophobes avec les composés et de groupements sulfoniques, responsables d'échanges de cations (17). L'OASIS $^{\circledR}$ est composée d'une phase responsable d'interactions hydrophobes et hydrophiles avec les composés et également de groupements sulfoniques.

Dans le cas de la zopiclone, le rendement de l'ELL, la technique de routine du laboratoire est très faible. Le

Tableau $\boldsymbol{V}$ : Rendements d'extraction des anti-inflammatoires non stérö̈diens.

\begin{tabular}{|c|c|c|c|c|c|c|c|c|c|}
\hline \multirow{2}{*}{$\begin{array}{l}\text { Anti- } \\
\text { inflammatoires } \\
\text { non stéroïdiens }\end{array}$} & \multicolumn{3}{|c|}{ ELL } & \multicolumn{3}{|c|}{ OASIS, fraction acide } & \multicolumn{3}{|c|}{$\mathrm{BEC}$, fraction acide } \\
\hline & $\mathrm{M} \%$ & $(\mathrm{ESM})$ & $\mathrm{CV}$ & M\% & (ESM) & $\mathrm{CV}$ & M \% & $(\mathrm{ESM})$ & $\mathrm{CV}$ \\
\hline Acide niflumique & $80^{0 . B}$ & (2) & 5 & 39 ${ }^{\mathrm{L.B}}$ & (4) & 21 & $\mathbf{0}^{\mathrm{O} . \mathrm{L}}$ & (0) & 0 \\
\hline Diclofénac & $85^{0 . B}$ & (4) & 10 & $52^{\mathrm{L} . \mathrm{B}}$ & (11) & 41 & $18^{0 . \mathrm{L}}$ & (1) & 13 \\
\hline Diflunisal & $76^{0 . B}$ & (3) & 8 & $30^{L . B}$ & (3) & 17 & $3^{\text {O.L }}$ & (1) & 67 \\
\hline Fénoprofène & $89^{\text {O.B }}$ & (2) & 6 & $\mathbf{5 0}^{\mathrm{L.B}}$ & (4) & 14 & $4^{\mathrm{O} . \mathrm{L}}$ & (1) & 68 \\
\hline Indométacine & 80 & (2) & 4 & 50 & (8) & 34 & 61 & $(16)$ & 53 \\
\hline Kétoprofène & $97^{\mathrm{O} . \mathrm{B}}$ & (3) & 7 & $52^{\mathrm{L} . \mathrm{B}}$ & (3) & 11 & $14^{0 . L}$ & (1) & 18 \\
\hline Naproxène & $89^{0 . B}$ & (2) & 5 & $23^{L . B}$ & (2) & 16 & $1^{\text {O.L }}$ & $(0)$ & 22 \\
\hline Piroxicam & $84^{\text {O.B }}$ & (3) & 8 & $0^{L . B}$ & (0) & 0 & $72^{\mathrm{O} . \mathrm{L}}$ & (3) & 8 \\
\hline
\end{tabular}

$\mathrm{L}$ : résultat significatif par rapport à la technique liquide-liquide.

$\mathrm{B}$ : résultat significatif par rapport à la technique BEC.

$\mathrm{O}$ : résultat significatif par rapport à la technique OASIS. 
Tableau VI : Rendements d'extraction des molécules diverses.

\begin{tabular}{|c|c|c|c|c|c|c|c|c|c|}
\hline \multirow{2}{*}{$\begin{array}{c}\text { Molécules } \\
\text { diverses }\end{array}$} & \multicolumn{3}{|c|}{ ELL } & \multicolumn{3}{|c|}{ OASIS, fraction basique } & \multicolumn{3}{|c|}{$\mathrm{BEC}$, fraction basique } \\
\hline & M\% & (ESM) & $\mathrm{CV}$ & M\% & (ESM) & $\mathrm{CV}$ & $\mathrm{M} \%$ & $(\mathrm{ESM})$ & $\mathrm{CV}$ \\
\hline Ambroxol & 97 & (3) & 7 & 83 & (4) & 11 & 85 & (9) & 20 \\
\hline Buflomédil & 89 & (3) & 7 & 94 & (2) & 5 & 94 & (3) & 6 \\
\hline Dilthiazem & $71^{\text {B.o }}$ & (4) & 13 & $9^{\mathrm{L}}$ & (1) & 2 & $89^{L}$ & (3) & 7 \\
\hline Hydroquinidine & 82 & (2) & 5 & 94 & (2) & 4 & 86 & (10) & 23 \\
\hline Hydroxyzine & 94 & (1) & 2 & 85 & (4) & 10 & 83 & (7) & 17 \\
\hline Lidocaïne & 77 & (11) & 27 & 83 & (6) & 15 & 89 & (6) & 13 \\
\hline Méfloquine & 77 & (5) & 12 & 96 & (1) & 3 & 73 & (15) & 42 \\
\hline Métoprolol & 73 & (12) & 32 & 69 & (4) & 11 & 65 & (4) & 11 \\
\hline Naftidrofuryl & 81 & (10) & 23 & 84 & (4) & 10 & 83 & (6) & 13 \\
\hline Noramidopyrine & 50 & (7) & 27 & 49 & (6) & 26 & 35 & (5) & 29 \\
\hline Piribédil & 92 & (2) & 5 & 98 & (2) & 7 & 92 & (3) & 6 \\
\hline Proguanil & $40^{\text {B.O }}$ & (2) & 9 & $\mathbf{0}^{\mathbf{L . B}}$ & (0) & 0 & $72^{\mathrm{L} . \mathrm{O}}$ & (7) & 20 \\
\hline Propranolol & 83 & (3) & 8 & $91^{\mathrm{B}}$ & (2) & 3 & $75^{\circ}$ & (6) & 16 \\
\hline
\end{tabular}

$\mathrm{L}:$ résultat significatif par rapport à la technique liquide-liquide.

$\mathrm{B}$ : résultat significatif par rapport à la technique BEC.

$\mathrm{O}$ : résultat significatif par rapport à la technique OASIS.

pKa de cette molécule est de 6,7. Le pH de l'extraction liquide-liquide $(\mathrm{pH}=9)$ est donc mal adapté, la zopiclone se trouvant essentiellement sous forme ionisée.

Les AINS sont bien extraits par l'ELL et mal extraits par les extractions solide-liquide. Ces molécules ne sont peut-être pas retenues sur les deux colonnes utilisées. Pour vérifier cette hypothèse, il serait possible de récupérer la fraction de sérum sortant de la colonne et de la ré-extraire par la technique ELL pour vérifier la présence d'AINS. Une autre possibilité est que ces molécules soient éluées au cours des étapes de lavage mais les différentes fractions de lavage dans les deux techniques ont été analysées et aucune trace de molécules n'a été détectée. Enfin, ces AINS peuvent également rester sur la colonne, sans être élués. Dans ce cas, il faudrait revoir les protocoles d'extraction en les adaptant à cette classe pharmacologique (en modifiant les $\mathrm{pH}$, les solvants d'élution, par exemple).

Les concentrations thérapeutiques de ces molécules sont importantes (proche de la dizaine de $\mathrm{mg} / \mathrm{l}$ ) permettant leur détection, malgré un rendement d'extraction faible. La répétabilité des extractions solide-liquide étant mauvaise, leur quantification par ces techniques reste difficile.

La technique OASIS ${ }^{\circledR}$ permet d'extraire l'ensemble des molécules basiques et neutres étudiées avec des rende- ments souvent équivalents voir meilleurs que la technique liquide-liquide, technique utilisée en routine dans notre laboratoire. Elle permet également l'extraction de molécules acides. Les molécules acides testées dans ce travail sont mal extraites par la colonne OASIS ${ }^{\circledR}$ mais l'étude devra être approfondie pour connaître la cause exacte de ces résultats médiocres et pour élargir l'application à d'autres molécules aussi bien acides que basiques et neutres.

La colonne OASIS ${ }^{\circledR}$ possède d'autres avantages pratiques. Elle permet l'extraction de molécules acides, neutres et basiques à partir de un $\mathrm{ml}$ de sérum alors que dans l'ELL, $2 \mathrm{ml}$ sont nécessaires. Elle permet l'utilisation d'un moindre volume de solvant par rapport aux deux autres techniques.

Enfin, La colonne OASIS ${ }^{\circledR}$ permet une extraction beaucoup plus rapide et plus reproductible que la BEC car le séchage de la colonne avant le dépôt de l'échantillon n'affecte pas le rendement ni la reproductibilité d'extraction et il n'est pas nécessaire de sécher la colonne avant l'élution (22). La durée d'extraction est un critère important : il faut compter 30 minutes pour 10 tubes avec les techniques liquide-liquide et $\mathrm{OASIS}^{\circledR}$, et 1 heure avec la BEC. Ce gain de temps est important dans le cadre d'urgences. 


\section{Conclusion}

L'objectif de ce travail était de développer une méthode de dépistage toxicologique large de xénobiotiques médicamenteux. Pour cela, trois extractions ont été comparées : extraction liquide-liquide (technique de routine), extraction solide-liquide sur colonne de silice BOND ELUT CERTIFY ${ }^{\circledR}$ (BEC) et sur colonne de copolymère (OASIS ${ }^{\circledR}$ MCX). La colonne OASIS $^{\circledR}$ a fourni dans l'ensemble les rendements d'extraction les plus importants excepté pour les anti-inflammatoires non stéroïdiens. Mais les AINS ont des concentrations thérapeutiques importantes permettant leur détection. La colonne OASIS ${ }^{\circledR}$ permet l'extraction de molécules basiques, acides et neutres en utilisant une seule colonne et un $\mathrm{ml}$ de sérum. Cette méthode est plus rapide, plus reproductible et permet une moindre utilisation de solvants que la BEC et l'ELL. La colonne OASIS ${ }^{\circledR}$ semble donc bien adaptée au dépistage toxicologique large à partir de sérum dans un cadre d'urgence, d'autant plus que cette technique pourrait être automatisée.

\section{Remerciements}

Nous tenons à remercier vivement l'équipe des techniciens du laboratoire de biochimie D et le P-Tox Group de l'hôpital Edouard Herriot pour l'aide qu'ils ont apportée à la réalisation de ce travail.

\section{Références}

1. Galliot-Guilley M., Fompeydie D. Dosage des médicaments pour le diagnostic et la surveillance des intoxications aigües. Rev. Prat. 1997 ; 47 : 721-725.

2. Lheureux P., Maes V., Askenasi R. Du bon usage du laboratoire de toxicologie. Premiere partie: aspects techniques. Rean. Urg. 1996 ; 5 (2) : 87-95.

3. Baud F.J. L'analyse toxicologique en urgence. JEUR $1989 ; 70-72$.

4. Lai C.K., Lee.T., Au K.M., Chan A. Y. W. Uniform solidphase extraction procedure for toxicological drug screening in serum and urine by HPLC with photodiode-array detection. Clin. Chem. 1997 ; 43 (2) : 312-325.

5. Lambert W.E., Meyer E., De Leenheet A. P. Systematic toxicological analysis of basic drugs by gradient elution of an alumina-based HPLC packing materiel under alkaline conditions. J. Anal. Toxicol. 1995 ; 19 : 73-78.

6. Logan B.K., Stafford D.T. Rapid screening for 100 basic drugs and metabolites in urine using cation exchange solid-phase extraction and high-performance liquid chromatography with photodiode-array detection. J. Anal. Toxicol. $1990 ; 14: 154-159$.

7. Tracqui A. Développement d'une méthode originale de screening toxicologique par chromatographie liquide haute performance avec détection par barrette de diodes (HPLC/DAD). Applications à la toxicologie clinique et médico-égale. Thèse pour l'obtention du doctorat 1993.
8. Turcant A., Premel-Cabic A., Cailleux A., Allain P Toxicological screening of drugs by microbore high-performance liquid chromatography with photodiode-array detection and ultraviolet spectral library searches. Clin. Chem. 1991 ; 37 (7) : 1210-1215.

9. Ojanpera I., Rasanen L., Vuori E. Automated quantitative screening for acidic and neutral drugs in whole blood by dual-column capillary gas chromatography. J. Anal. Toxicol. $1991 ; 15: 204-208$.

10. Puopolo P.R., Pothier M.E., Volpicelli S.A., Flood J.G. Single procedure for detection, confirmation and quantification of benzodiazepines in serum by liquid chromatography with photodiode-array detection. Clin. Chem. 1991 ; 701-706.

11. Puopolo P.R, Volpicelli S.A., Mackeen Johnson D., Flood J.G. Emergency toxicology testing (detection, confirmation and quantification) of basic drugs in serum by liquid chromatography with photodiode array detection. Clin. Chem. 1991; 37/12 : 2124-2130.

12. Tracqui A., Kintz P., Kreissig P., Mangin P. A simple and rapid method for toxicological screening of 25 antidepressants in blood or urine using high performance liquid chromatography with photodiode array detection. Ann. Biol. Clin. 1992 ; 50 : 639-647.

13. Demedts P., Wauters F., Neels H. Evaluation of the Remedi profiling system. Eur. J. Clin. Chem. Biochem. $1994 ; 34: 409-417$.

14. Guitton J., David O., Mialon A., Manchon M. Evaluation d'un automate de chromatographie liquide permettant un screening toxicologique dans les liquides biologiques. Ann. Biol. Clin. 1993; 51 : 611-617.

15. Kalasinsky K. S., Schaefer T. Forensic application of an automated drug profiling system. J. Anal. Toxicol. 1995 ; $19: 412-418$.

16. Manchon M., Mialon A., Berny C., Baltassat P. Intérêt de l'automate de chromatographie Remedi pour le diagnostic analytique des intoxications médicamenteuses.Ann. Biol. Clin. 1997 ; 55 : 223-228.

17. Chen X.H., Franke J.P., Ensing K., Wijsbeek J., De Zeeuw R.A. A single-column procedure on Bond Elut Certify for systematic toxicological analysis of drugs in plasma and urine. J. Forensic Sci. 1991 ; 61-71.

18. Drummer O.H., Kotsos A., Mcintyre L.M. A class-independent drug screen in forensic toxicology using a photodiode array detector. J. Anal. Toxicol. $1993 ; 17: 225$ 229.

19. Koves E.M. Use of high liquid chromatography-diode array detection in forensic toxicology. J. Chromatogr. A. 1995 ; 262 : 103-119.

20. Caporal-Gauthier J., Nivet J.M., Algranti P., Guilloteau M., Histe M., Lallier M., N'Guyen-Huu J.J., Russotto R. Guide de validation analytique, rapport d'une commission SFSTP (Méthodologie). STP Pharma pratiques $1992 ; 2$ (4) : 205-226.

21. Vassault A., Azzedine M.C., Bailly M., Cam G., Dumont G., Erkinjian O.G., Feldmann D., Georges P., Gerhardt N.F., Goudard M., Grafmeyer D., Henny J., Mathieu M., Mollard J.F., Naaudin C., Trepo D. Comité scientifique, section de standardisation, commission de validation technique. 1985 ISB, 11, 1.

22. Gaillard Y. Analyse des xénobiotiques après extraction en phase solide en pharmacotoxicologie. Applications cliniques, médico-légales et sportives. Thèse pour le diplôme de doctorat $1999 ; 122-99$. 